\title{
El actor de la lucha no violenta en Ecuador: El rol de los jóvenes en las elecciones presidenciales 2017 en Ecuador
}

\section{The actors of the non-violent struggle in Ecuador: The role of young people in the 2017 presidential elections in Ecuador}

\author{
Paola Verónica Lozada Lara \\ Pontificia Universidad Católica del Ecuador, Ecuador
}

Autor de correspondencia: pvlozada@puce.edu.ec

Fecha de recepción: 12 de junio de 2017 - Fecha de aceptación: 30 de noviembre de 2017

\section{Resumen}

Durante el gobierno del presidente Rafael Correa, la población ecuatoriana ha estado constantemente inmersa en luchas no violentas cuando las medidas del gobierno distaron de los planteamientos iniciales de la Revolución Ciudadana o cuando sus intereses se vieron afectados. En las elecciones presidenciales del 2017 en Ecuador se organizó una campaña no violenta tendiente a transparentar los resultados debido a las irregularidades del proceso. En esta lucha se destaca el rol de un sinnúmero de actores como: militares, iglesia, medios de comunicación, figuras políticas, entre otros; no obstante, la participación juvenil ha sido desapercibida. De ahí que esta investigación pretende visibilizar a este grupo poblacional, específicamente en lo que concierne a la frecuencia, motivaciones, mecanismos de participación, nivel de organización y conocimientos sobre no violencia, a partir de un estudio exploratorio realizado en 125 jóvenes universitarios comprendidos entre los 18 y 24 años de edad en la ciudad de Quito.

Palabras claves: lucha no violenta; jóvenes; elecciones presidenciales; Ecuador

\begin{abstract}
During the government of President Rafael Correa, the Ecuadorian population had been constantly involved in nonviolent struggles, either when government measures diverged from the initial approaches of the Citizen Revolution or when their interests were affected. In 2017 presidential elections in Ecuador, a non-violent campaign was organized in order to achieve clean elections due to irregularities in the process. In this struggle, the role of actors such as: military, church, media outlets, political figures, among others, is highlighted. However, youth participation has been overlooked. Hence, the purpose of this research is to give visibility to this population group, focusing on the frequency, motivations, mechanisms of participation, level of organization and knowledge about non-violence. This research is based on an exploratory study of 125 university students between 18 and 24 years old in Quito city.
\end{abstract}

Key words: nonviolent struggle; youth; presidential elections; Ecuador 


\section{Introducción}

Esta investigación pretende revisar la lucha no violenta en el gobierno de Rafael Correa y de manera particular la participación de los jóvenes universitarios durante el proceso de las elecciones presidenciales en Ecuador en el año 2017. La primera parte del estudio consiste en una revisión de los principales momentos en que se evocó la no violencia durante el gobierno de Rafael Correa y los mecanismos no violentos utilizados por diferentes actores. La segunda parte detalla la lucha no violenta en la primera y segunda ronda del proceso electoral, actores, mecanismos y resultados. El tercer segmento se suscribe estrictamente al rol de uno de los actores menos considerados en la organización de luchas no violentas: los jóvenes.

Esta investigación toma como base a varios de los autores más relevantes sobre no violencia como: Robert Helvey, Kurt Schock y Christopher Miller. El primero, Helvey (2004) con su obra: "On Strategic Nonviolent Conflict: Thinking about the Fundamentals", se enfoca en los fundamentos de la lucha no violenta como una opción probada como viable y efectiva en la consecusión de cambios políticos reduciendo los daños colaterales, para ello alienta una planificación estratégica acorde a la realidad de cada conflicto y su aplicación en causas particulares. Schock (2005) en su libro: "In Unarmed Insurrections: People Power Movements in Non democracies" enfatiza la idoneidad de la no violencia frente a la violencia a través de la casuística, profundiza el análisis entre la teoría y la práctica identificando las razones del éxito o fracaso. Por último, en el caso de Miller (2006a y 2006b) se han elegido dos de sus obras, la una "In Strategic Nonviolent Struggle: A Training Manual" se traduce en una guía práctica para el ejercicio de la no violencia y la transformación pacífica de conflictos, mediante una comprensión amplia de las bases, el contexto, las acciones y los mecanismos específicos tendientes a librar luchas no violentas cuando el caso lo amerite. La otra obra citada en esta investigación "Only Young Once: An Introduction to Nonviolent Struggle for Youths" combina las estrategias no violentas con la participación de los jóvenes. Cabe mencionar que ambos libros de Miller fueron diseñados para responder a las necesidades de la Región Delta del Niger.

La necesidad y novedad de este estudio radica en la tendencia mostrada por la población ecuatoriana hacia la no violencia, lo cual se constata a través de la aplicación de un sinnúmero de mecanismos no violentos como las protestas, marchas, plantones, vigilias, actos simbólicos, entre otros, a lo largo de los 10 años del gobierno de Correa. Durante las elecciones presidenciales del 2017, el movimiento no violento se fortaleció al concentrarse en la campaña para transparentar los resultados de la primera y segunda vuelta electoral. Esta iniciativa logró acoger cada vez a más actores; no obstante, uno de los pilares de soporte que no ha sido visibilizado son los jóvenes; de ahí que se pretenda revisar la participación de este grupo poblacional, específicamente de los jóvenes universitarios entre 18 y 24 años en la ciudad de Quito.

La hipótesis planteada en este estudio es la siguiente: los jóvenes constituyen un importante pilar de soporte en las luchas no violentas; no obstante, su participación ha sido opacada durante las elecciones presidenciales del 2017 en Ecuador para resaltar el rol de los actores que tradicionalmente detentan mayor poder. 


\section{Método}

El presente estudio se orientó a jóvenes universitarios de la ciudad de Quito, comprendidos entre 18 y 24 años de edad. El rango de edad elegido corresponde a la edad promedio de la vida estudiantil universitaria de conformidad con el Sistema Integrado de Conocimiento y Estadística Social del Ecuador (SICES), mientras que la delimitación espacial (Quito) se debe a la importancia de estudiar este fenómeno en la ciudad capital del Ecuador.

Para ello se elaboró una encuesta de 12 preguntas orientada a obtener información acerca del conocimiento que los jóvenes tienen sobre no violencia, los mecanismos, la importancia, frecuencia y razones de su participación, así como también el nivel de organización de la lucha no violenta protagonizada durante las elecciones. Esta encuesta fue respondida por un total de 128 personas, de las cuales 125 personas cumplían con el perfil especificado, por lo que los resultados tabulados corresponden a exclusivamente a los 125 estudiantes.

El estudio responde a un tipo de investigación exploratoria, por tratarse las elecciones presidenciales de un tema relativamente reciente, ha sido poco estudiado; mientras que la participación de los jóvenes en las luchas no violentas en Ecuador no ha sido abordada.

\section{Desarrollo}

\section{Manifestaciones no violentas en el gobierno de Rafael Correa}

El presente apartado adopta una de las premisas de Schock (2005) acerca de que la lucha no violenta requiere paciencia para lograr cambios estructurales. En ese sentido se toma como punto de partida la elección de Rafael Correa como Presidente de la República en el 2006 para repasar algunos de los principales problemas que han evocado resistencia civil.

La Revolución Ciudadana promulgada por Correa prometía cambios estructurales en la administración estatal y en el marco normativo, así como una sociedad más equitativa. A nivel de la administración pública se evidenció una restructura y fortalecimiento del aparato estatal con el establecimiento de cinco funciones (ejecutivo, legislativo, judicial, electoral y poder ciudadano) y la creación de nuevas entidades como Ministerios Coordinadores, institutos, secretarías técnicas y nacionales, entre otros. A nivel normativo destaca la nueva constitución del 2008 y con ella un sinnúmero de cuerpos jurídicos instaurados en códigos, leyes y decretos ejecutivos; mientras que a nivel social se encuentra el ideal de la equidad social fundamentada en el Socialismo del Siglo XXI (Movimiento País, 2014).

No obstante, durante los 10 años de gobierno de Correa se han encontrado: funciones del estado e instituciones cuyas cabezas están alineadas al régimen como en el caso de la Fiscalía General de la Nación ${ }^{1}$ y el Consejo Nacional Electoral (CNE) ${ }^{2}$ respectivamente, leyes que

\footnotetext{
${ }^{1}$ Galo Chiriboga ocupó el cargo de Ministro de Minas y Petróleos al inicio del gobierno de Correa (2007), abogado en el juicio Correa sostuvo contra el Banco Pichincha y en el 2011 fue elegido Fiscal General de la Nación. ("Galo Chiriboga", 2011, mayo 3).

2 Según Basabe y Martínez (2014), el CNE estaba presidido por colaboradores cercanos al gobierno lo cual hace que la imparcialidad de esa institución sea cuestionable.
} 
restringen las garantías y libertades como la Ley de comunicación y la Ley de Aguas, así como pruebas de corrupción, para citar algunas: sobreprecios en obras de infraestructura, videos mostrando la manipulación de bonos de la deuda en Wikileaks (cable 109333) ${ }^{3}$, negociaciones bajo la mesa realizadas por altas autoridades y denunciadas por ex ministros ${ }^{4} \mathrm{y}$ sobornos descubiertos por el Departamento de Justicia de Estados Unidos en el caso Odebrecht. ${ }^{5}$ En ese contexto, la población ecuatoriana ha rechazado medidas del gobierno cuando sus intereses se han visto afectados o cuando dichas medidas distan de los planteamientos iniciales de la Revolución Ciudadana. Uno de los actores más representativos de esa lucha son los indígenas, quienes a través protestas, marchas y manifestaciones han plasmado su rechazo a las actividades mineras y la ley de Aguas del 2009 al 2011, llegando a la presentación de una denuncia por la criminalización de la protesta ante el FIDH el $20126^{6}$.

Los medios de comunicación constituyen otro actor clave en la lucha no violenta. Particularmente con respecto a la Ley de Comunicación, periodistas nacionales y organizaciones internacionales defensoras de derechos humanos han estado presentes a través de la publicación de caricaturas, artículos, editoriales, libros e informes; así como a través del activismo virtual en redes sociales. Algunos ejemplos son: las caricaturas de Adrián Bonilla "Bonil”, artículos de Andrés Ortiz y Hernán Reyes, el libro Silencio Asfixiante de Fundamedios, informes de la Comisión Interamericana de Derechos Humanos y Human Right Watch, entre otros; mientras que a nivel de activismo virtual sobresalen las cuentas de Facebook como Crudo Ecuador y de Tweeter como Levántate Ecuador y ResistenciaEC; así también las publicaciones de periodismo investigativo de Cuatro Pelagatos y Plan V.

En cuanto a grupos de la sociedad civil se puede mencionar al colectivo Yasunidos, quien lideró la protesta para impedir la explotación hidrocarburífera del Parque Nacional Yasuní en el 2014, una vez que falló la iniciativa del gobierno encaminada a recabar los fondos necesarios para mantener el petróleo bajo tierra. ${ }^{7}$ Este caso combinó algunos de los mecanismos no violentos antes mencionados como las marchas, plantones, activismo virtual e incluyó otros nuevos como actos públicos simbólicos entre los que destacan la pintura y vestimenta como protesta y la petición masiva de un referendo a través de recolección de firmas. No obstante, el colectivo y asumidos, según el CNE, no consiguió el número de firmas necesarias para llevar a cabo su propósito. (Mena, P., 2014, abril 14)

\footnotetext{
${ }^{3}$ Este evento se conoció como "pativideos” en virtud del ex Ministro de Economía, Ricardo Patiño. En los videos se identificaba a Patiño conversando con otro ex Ministro de Economía acerca de la posibilidad de obtener USD 50 millones asustando el mercado. ("Pativideo", 2011, abril 30).

${ }^{4}$ El ex ministro de Hidrocarburos durante el gobierno de Correa, Carlos Pareja Yannuzzelli, denunció a través de redes sociales (CAPAYA leaks) que las negociaciones encubiertas en Petroecuador serían aprobadas por el vicepresidente, Jorge Glas. (“Aquí todas las revelaciones”, 2017, febrero 3).

${ }^{5}$ De acuerdo al Departamento de Justicia de los Estados Unidos Odebrecht pagó USD 33.5 millones a funcionarios y políticos ecuatorianos del 2007 al 2016. (Megale,Matosso, Wiziack \& Valente, 2016).

${ }^{6}$ La protesta indígena constituye además un ejemplo de la evolución de los mecanismos de protesta desde manifestaciones y bloqueo de carreteras en los noventa, incluyendo su intervención en el derrocamiento de presidentes como Bucaram (1997) y Mahuad (2000) hasta la cooptación de espacios de poder e inserción del concepto de plurinacionalidad en la Constitución del 2008.

${ }^{7}$ La Iniciativa Yasuní ITT pretendió recabar USD\$ 3.6 billones de la comunidad internacional, sin embargo, el gobierno alcanzó apenas el $0.37 \%$ del total.
} 
En adelante el activismo virtual se extendió posiblemente a la par con el uso de redes en el Ecuador. De acuerdo a un informe publicado por el Instituto Nacional de Estadísticas y Censos (INEC) en el 2015, la población de 12 y más años que utilizaba redes sociales ascendía a 41.5\%, esto es un total de 4.995.474 usuarios (García, J., 2015). De ahí que se entienda que tres proyectos de ley presentados por el gobierno para incrementar impuestos tuvieron como respuesta social manifestaciones auto convocadas por redes sociales que duraron un mes en junio del 2015 y más de una semana en abril del 2016. (Ospina, 2015).

En suma, las protestas sociales durante el gobierno de Rafael Correa han sido constantes, encontrando diferentes rostros (pese a que no se visibiliza la participación juvenil) y aplicando mecanismos distintos. Aún si en los casos planteados no ha habido resultados favorables, sí se evidencia que la población ecuatoriana ha permanecido activa en su lucha no violenta, lo cual confirma no sólo la necesidad de paciencia según Schock (2005) sino además constancia para lograr cambios estructurales.

\section{Resistencia civil en las elecciones presidenciales del 2017}

Este apartado se basa en la premisa de Gen Sharp (2005) de que la no violencia está identificada por lo que gente hace y no por lo que piensa. Para ello se revisa el caso más reciente que evocó protestas sociales, las elecciones presidenciales del 2017, tanto la primera como la segunda vuelta. Este estudio se limitará a los dos candidatos más opcionados, Lenín Moreno y Guillermo Lasso.

Las propuestas políticas de ambos candidatos difieren radicalmente en contenido e ideología. Por una parte, Moreno con su movimiento Alianza País busca continuar con los planteamientos del gobierno de Rafael Correa; y, extender la Revolución hacia la ética, el conocimiento, la juventud, la política, entre otros; para ello se prioriza el empleo, préstamos productivos, acceso a la educación superior y evitar la monopolización de los medios (Movimiento País, 2016). Por otra parte, Lasso, un ex banquero y empresario aliado a la derecha, propuso desde la alianza política Creo-Suma, la creación de un millón de puestos de trabajo, eliminación de impuestos para incentivar la inversión extranjera, firmar acuerdos de cooperación con Estados Unidos, China, Japón y la Alianza del Pacífico, derogar la ley de comunicación, vender los medios de comunicación estatales y restablecer la democracia a través de la separación de poderes ("Apertura a los acuerdos", 2017, enero 10).

Las elecciones presidenciales tuvieron lugar el domingo 19 de febrero de 2017. En el contexto institucional, días anteriores el CNE (2017, enero 10) informó que los resultados preliminares estarían listos el mismo día a las 20:00 horas, mientras que los resultados oficiales se presentarían 10 días después. A nivel normativo, de acuerdo a la Ley Orgánica Electoral y de Organizaciones Políticas de la República del Ecuador Código de la Democracia (2009), un candidato requiere el $50 \%$ de los votos más uno o al menos el $40 \%$ y una diferencia de diez puntos porcentuales sobre el segundo candidato para ganar las elecciones. En el nivel social, los opositores del gobierno advertían sobre la posibilidad de fraude; de ahí que se publicaron vía redes sociales varias recomendaciones como: llevar un esfero no borrable de color azul el día de elecciones, usar camisetas amarillas o de la selección ecuatoriana para identificar el voto contrario al gobierno, documentar cualquier inconveniente durante el proceso electoral y acudir 
al CNE en caso de evidente fraude. Adicionalmente, la alianza política Creo - Suma reclutó entre adherentes y simpatizantes a más de 40.000 Voluntarios para monitorear las elecciones (“Lasso presentó red de control electoral”, 2017, febrero 14).

El día de las elecciones, la ONG Participación Ciudadana, entidad acreditada por el CNE para realizar un exit poll, anunció a las 21:00 horas la posibilidad de una segunda vuelta en base al $87.4 \%$ de la muestra admitida y $1 \%$ de margen de error (Participación Ciudadana, 2017). A las 23:45 horas el CNE apagó la pantalla que mostraba los resultados y cerró el principal centro de transmisión sin explicaciones; a ese momento se mostraba un total de $80.3 \%$ de actas escrutadas que daban como resultado 38.9\% para Lenín Moreno y 28.5\% Guillermo Lasso ("CNE cerró centro de transmisión”, 2017, febrero 20). Tres días después el CNE confirmó la necesidad de una segunda vuelta que tendría lugar el 2 de abril.

Durante la espera de los resultados oficiales se desplegó una campaña no violenta autoconvocada por la misma población en las afueras del CNE, la cual bloqueó importantes calles de la capital, para protestar de forma masiva contra la falta de transparencia del proceso electoral y solicitando resultados inmediatos. El movimiento no violento crecía en número y fuerza en distintas ciudades del país (Guayaquil, Cuenca, Ambato) e incluyó a la mayor parte de los pilares de soporte referidos por Christopher Miller (2006a) en su "Strategic nonviolent struggle: A training manual". De acuerdo a este autor, los pilares de soporte son aquellas instituciones $\mathrm{u}$ organizaciones de soporte que permiten mantener y ejercer el poder (Miller, 2006a). Algunos pilares constantes son la policía, los militares, la iglesia, comunidades de negocios, los medios. En el caso ecuatoriano tanto el Consejo de Generales del Ejército (2017), la Conferencia Episcopal Ecuatoriana y el Consejo Nacional de Cámaras realizaron similares planteamientos sobre garantizar la voluntad del pueblo y agilizar la presentación de resultados ("Fuerzas políticas", 2017, febrero 21); la Policía Nacional se pronunció sobre las denuncias de papeletas marcadas y señaló que todas ellas fueron enviadas al CNE (Romero, D., 2017, febrero 19); los medios nacionales cubrían cada detalle del proceso electoral mientras que la prensa internacional sugería la posibilidad de fraude. Así el diario El País de Colombia publicó: "Tensa situación política en Ecuador, ante denuncias de fraude en elecciones", el Diario de información y análisis de Intereconomía, La Gaceta, hizo lo propio bajo el titular: "Así se gestionó el fraude electoral en Ecuador", en el diario La Nación de Argentina se encontraba el artículo: "Elecciones con olor a fraude", en CNN es español se afirmaba "Hay evidencias de irregularidades en elecciones", mientras que el Centro Estratégico Latinoamericano de Geopolítica (CELAG) hablaba de "la Agenda del Fraude".

No obstante, según Miller (2006a) cada sociedad es única y cuenta con distintos pilares de soporte; de ahí que durante las elecciones en Ecuador se destacarían el movimiento indígena (Conaie, 2017), autoridades locales de Quito y Guayaquil; y, personalidades públicas como León Roldós, ex vicepresidente de la República, quienes solicitaron transparencia en el proceso electoral y la rápida publicación de resultados. Estos actores, que llamaban a la paz en aras de prevenir que la violencia se convierta en un contaminante del movimiento, han sido priorizados tanto a nivel político como mediático. No obstante, la participación de los jóvenes como un importante pilar en la sociedad ecuatoriana ha sido relegada o invisibilizada, a pesar que, de acuerdo con el INEC, en el Censo de Población y Vivienda de 2010, los jóvenes representaban el $13 \%$ de la población en el Ecuador, mientras que el 15.2\% de la población se encontraba 
cursando educación superior. Con base en el mismo Censo para la provincia de Pichincha la población de 15 a 19 años representó el $9.3 \%$ y de 20 a 24 años el 9.6\%. Los datos estadísticos más recientes con respecto a los estudiantes universitarios en Ecuador corresponden al Sistema Integrado de Conocimiento y Estadística Social del Ecuador (SICES, 2016) donde se indica que el número de estudiantes matriculados en educación superior corresponde al $30.4 \%$ de jóvenes entre 18 y 24 años.

Durante la primera vuelta electoral, la población ecuatoriana, incluidos los pilares de soporte, se organizaron en torno a un objetivo claro la transparencia del proceso y resultados inmediatos. En esas condiciones la campaña no violenta cumplió su propósito, esto es, se legitimó la presencia de dos candidatos, Moreno y Lasso como finalistas y se proclamó una segunda vuelta en la que se decidiría el futuro presidente del país para los próximos cuatro años. No obstante, el triunfo del movimiento no violento fue parcial pue se debía continuar con la lucha hasta la culminación del proceso electoral en medio de una población dividida entre dos candidatos, situación que conllevaba aún mayor complejidad en el resguardo del proceso democrático.

Después de la primera vuelta se produjo gran conmoción en el escenario político y social por cuanto varios de los pilares del poder fueron amedrentados o anulados. El Consejo de Generales de la Armada que apoyó la campaña no violenta fue retirado del poder; el gobierno de Correa cambió la cúpula militar ("El jefe saliente", 2017, mazo7). Entre las fíguras públicas, Flor María Palomeque, una comediante política conocida como "la Mofle" no pudo salir al aire después de haber mencionado la palabra "fraude" en un programa de televisión ("Palomeque", 2017, mazo 21). A nivel privado, la encuestadora Cedatos, que obtuvo los resultados más similares a los oficiales durante la primera ronda, recibió una denuncia por alteración de datos. Adicionalmente, Cedatos hizo denuncias públicas sobre hackeos a su página web y cuentas de correo electrónico, así como haber víctima de amenazas (Cedatos, 2017, abril 5). Finalmente, días antes de la segunda vuelta la Policía Nacional cercó los alrededores del CNE en Quito.

Sin embargo, el evento que provocó mayor controversia fueron los ataques perpetuados contra Guillermo Lasso y su familia a la salida de un partido de fútbol de la selección nacional. ${ }^{8} \mathrm{Al}$ respecto, las redes sociales mostraban personas a favor de lo sucedido, mientras que otras llamaban a la paz ("Lasso", 2017, marzo 28). La división entre la población ecuatoriana se había exacerbado e incluso peor, la violencia parecería justificarse.

Los opositores al gobierno nuevamente alertaron sobre la posibilidad de fraude. Sin embargo, a pesar de las medidas adoptadas como los voluntarios para monitorear las elecciones y las fotografías a las actas electorales, se encontraron inconsistencias en el proceso electoral.

El día de las elecciones, 2 de abril, tuvo como principal característica la incertidumbre. Al inicio del conteo Lasso estaba a la cabeza; sin embargo, la página web del CNE tuvo inconvenientes durante veinte minutos y cuando volvió a la normalidad, los resultados arrojaban

\footnotetext{
${ }^{8}$ De manera posterior a este evento se verificó que una gran cantidad de tickets fueron adquiridos por el gobierno y pagados con fondos de un banco estatal abierto al público. De acuerdo a las declaraciones de la empresa comercializadora de tickets, el día del partido el estadio no contaba con la seguridad acostumbrada en virtud de un pedido expreso de entidades del gobierno, las cuales asumirían esa responsabilidad. (Pallares, M., 2017, marzo 20)
} 
que Moreno lideraba el proceso. Más tarde el CNE aclaró la diferencia entre el sistema de escrutinios y la web de difusión de resultados (Noboa, A., 2017, abril 3); no obstante, la desconfianza crecía.

Al final de la jornada electoral, los ecuatorianos contaban con cuatro diferentes resultados de los exit polls. Cedatos (2017) mostró a Lasso como el ganador con 53.02\% y Moreno con 46,98\%. Perfiles de Opinión, una encuestadora cercana al gobierno, identificó a Moreno como el nuevo presidente con $52.2 \%$ contra 47,8\% para Lasso ("Exit poll”, 2017, abril 2). Más tarde, el CNE proclamó a Lenín Moreno como el nuevo presidente con 51.15\% mientras que Guillermo Lasso obtuvo 48.85\%. Participación Ciudadana (2017, abril 2), la ONG que tuvo un rol clave durante la primera vuelta, indicó que había empate técnico. Tal variedad de resultados motivó mayores dudas sobre la fidelidad y transparencia del proceso electoral.

Como consecuencia, la población ecuatoriana nuevamente acudió a las calles. La presencia de personas que apoyaban a ambos candidatos revelaría mayor división social y disminución de la confianza en las autoridades e instituciones públicas. Pese a ello, la campaña no violenta incluyó una vigilia permanente, concentración en las oficinas del CNE de las principales ciudades del país (Quito, Guayaquil, Cuenca, Ambato), sonido de pitos de la bocina de autos, así como bloqueo de calles, túneles y puentes para mostrar su inconformidad ("Expectativa, 2017, abril 6). Nuevamente las redes sociales fueron el mecanismo más poderoso para canalizar las demandas ciudadanas y publicar las inconsistencias como por ejemplo actas adulteradas. La población solicitaba el reconteo de votos.

El 4 de abril, el presidente del CNE confirmó mediante cadena nacional el triunfo de Lenín Moreno e indicó que los resultados eran irreversibles. La alianza política Creo-Suma presentó una demanda por fraude y se apoyó de 1795 actas que mostraban irregularidades ante las Cortes Provinciales Electorales de conformidad con la ley ("Creo denuncia", 2017, abril 5).

La presencia de los medios estaría limitada en virtud de las restricciones de la Ley de Comunicación. Los canales de televisión daban poca cobertura a la propuesta de reconteo; lo que contrastaba con el llamado de atención a una radio local por parte de la Superintendencia de la Información y Comunicación, por convocar a la ciudadanía a la revuelta, ignorar las decisiones legítimas de las autoridades, promover la xenofobia y pedir a la policía que intervenga ilegalmente en el proceso democrático (Ochoa, C., 2017, abril 5).

Adicionalmente, el 7 de abril de 2017, la policía judicial ordenó el allanamiento a las oficinas de Cedatos debido a la falsa información emitida; de acuerdo a Rafael Correa, la encuestadora había atacado la fe pública (“Oficinas de Cedatos”, 2017, abril 8). Al día siguiente, durante el enlace sabatino 518 (Presidencia de la República del Ecuador, 2017, abril 8), Correa fue categórico al señalar a Cedatos, la ONG Participación Ciudadana y los medios como los responsables de la difusión errónea de resultados electorales.

A pesar de la intimidación a periodistas y presentadores de televisión, amenazas a funcionarios de Cedatos y Participación ciudadana. La campaña no violenta comprometió a nuevos miembros a su causa: políticos, organizaciones indígenas y una Consejera del CNE quien apoyaba el reconteo de votos (“Consejera”, 2017, abril 5). El 5 de abril, Alianza País anunció su 
acuerdo con el reconteo, pero a diferencia de la solicitud de Creo - Suma, no para todos los votos.

Después de dos semanas de protestas continuas, el 18 de abril, el CNE organizó un reconteo público únicamente de las actas impugnadas tanto por Creo-Suma como Alianza País; esto es de un total de 1.275.450 votos ("Recuento de votos", 2017, abril 18). Aún si esta acción también fue criticada por los opositores al gobierno, se confirmó a Lenín Moreno como Presidente del Ecuador para el periodo 2017-2021. En estas condiciones, el nuevo gobernante no puede desconocer el poder de la no violencia, ni la voluntad e interés de la población por defender procesos democráticos, elementos que se encontrarían presentes en la nueva administración.

Pese a haberse mermado la confianza en las autoridades e instituciones, encontrar un país dividido e inclinarse por diferentes preferencias políticas o no tenerlas, estos elementos no constituyeron un impedimento para que la población se una a la campaña no violenta en aras de transparentar los resultados electorales; lo que evidencia que en el caso de las elecciones presidenciales en Ecuador la lucha no violenta se identifica por lo que la gente hace y no por lo que creen.

\section{Resultados y Discusión}

De acuerdo a Robert Helvey (2004) "una preocupación primaria de los regímenes autoritarios es prevenir que los jóvenes se politicen" a menos que esa politización "sea apoyada y controlada por el gobierno". En esta afirmación subyace la importancia de los jóvenes en las luchas no violentas; no obstante, una de las limitaciones que se resalta en este estudio consiste en la escasa atención prestada a la participación de los jóvenes durante el proceso de elecciones presidenciales en Ecuador. De ahí que esta investigación pretende visibilizar la participación de dicho grupo poblacional durante el despliegue de la campaña no violenta.

Como resultado de esta encuesta se encontró que más del $60 \%$ de los encuestados dice conocer vagamente sobre no violencia y apenas un $34.4 \%$ conoce a profundidad sobre el tema, lo que mostraría una falencia en la enseñanza y capacitación sobre la no violencia como una alternativa realista a la violencia entre los jóvenes.

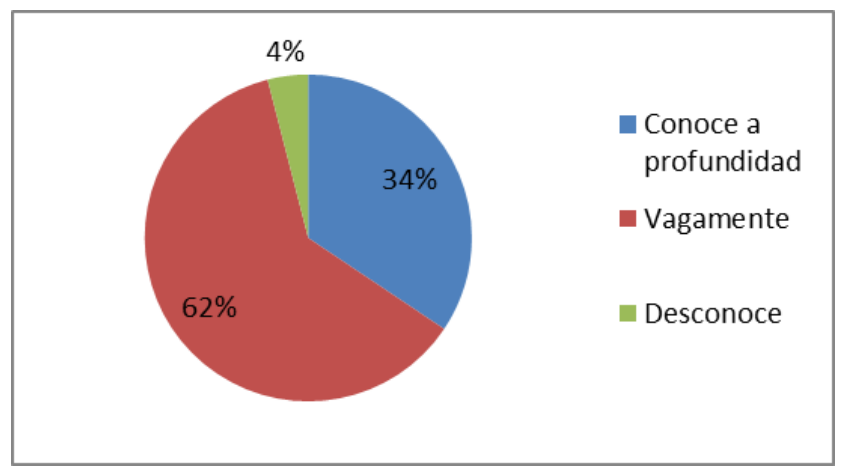

Gráfico 1: Conocimiento sobre no violencia Fuente: Encuesta sobre no violencia a estudiantes universitarios Elaborado por: Paola Lozada 
A la par con lo anterior, llama la atención que del total de encuestados únicamente el 34\% afirma haber participado en protestas sociales. Con ellos se procedió a analizar dos variables, la frecuencia y los mecanismos de participación. En relación a la frecuencia se conformaron tres rangos de opción, esto es de 1 a 3 participaciones, de 4 a 7 participaciones y de 8 a más. El rango con mayor porcentaje fue el primero, con el $83 \%$.

Sobre los mecanismos utilizados por los jóvenes, Miller (2006b) en su libro “Only Young Once: An Introduction to Nonviolent Struggle for Youths" indica que ellos empiezan por lo que conocen para iniciar acciones no violentas. Adicionalmente, los jóvenes se adaptan más a ciertos métodos que a otros, en algunos casos debido a que tienen más tiempo y energía, pero tienden a verse limitados en cuanto a legitimidad, habilidad, conocimiento y recursos materiales. En el caso ecuatoriano los mecanismos preferidos por los estudiantes universitarios encuestados son las marchas (30\%), a continuación, se muestra un empate entre los actos simbólicos y el activismo virtual (19\%), seguido de las protestas (17\%). La preferencia por las marchas podría explicarse por la recurrencia en el uso de este mecanismo durante el gobierno de Correa; el activismo virtual respondería a la masificación de las redes sociales, mientras que los actos simbólicos corresponderían a la necesidad de identificación con ciertos grupos en función de valores sociales.

Con la encuesta también se indagó acerca de la necesidad de participación activa por parte de los jóvenes, a la cual apenas el $10.57 \%$ dio una respuesta negativa, lo que contrasta con el $66 \%$ de personas que indicaron no haber participado en protestas sociales. Es decir, los encuestados están de acuerdo con que los jóvenes intervengan de manera más activa, pese a no haberse personalmente inmiscuido en las luchas sociales. En este punto los comentarios redundan alrededor de tres ejes: mayor información sobre los temas que aquejan a la sociedad, incentivar espacios para los jóvenes, mayor representación social.

Con respecto a las razones que motivan o motivarían a participar en protestas sociales, cabe la mención de Helvey (2004), quien que no se trata de lo que los jóvenes pueden perder sino de lo que pueden ganar en una sociedad justa y libre. Así pues, los resultados señalan que en primer lugar está la afinidad con los temas con $40.02 \%$, en segunda instancia los principios (32.4\%) y por último el cambio de esquemas (23.5\%).

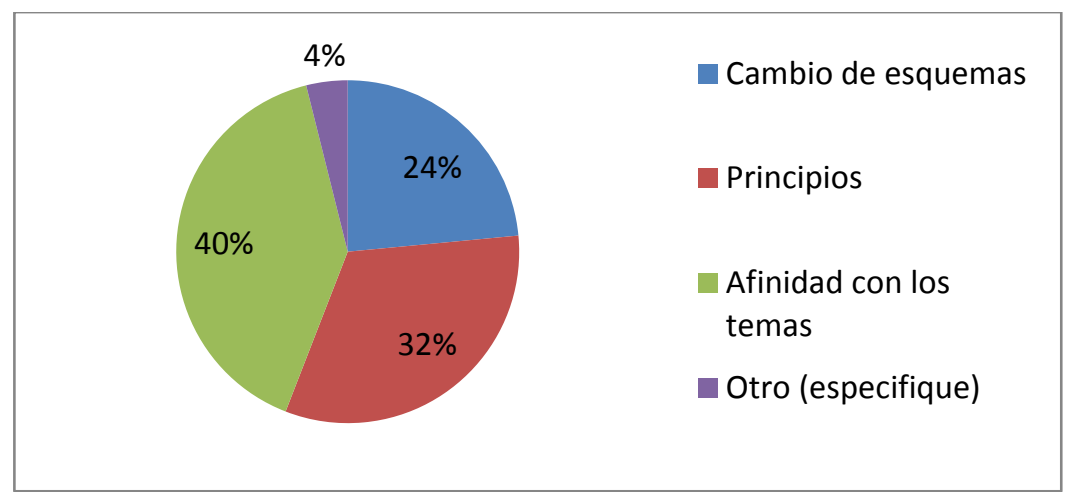

Gráfico 2: Motivos para unirse a protestas sociales Fuente: Encuesta sobre no violencia a estudiantes universitarios Elaborado por: Paola Lozada 
En lo que respecta a la campaña no violenta durante las elecciones presidenciales se encontró similares porcentajes de participación, esto es apenas un tercio de las personas encuestadas (34\%) se unieron a la campaña por la transparencia. Los resultados sobre los mecanismos más utilizados también se repiten en las elecciones presidenciales, pues se ubican de manera prioritaria las marchas, el activismo virtual y los simbolismos; sobre este último resaltan prendas de vestir, banderas y sonar la bocina de los autos. La equivalencia en los resultados sobre la participación de los jóvenes en luchas sociales con respecto a su participación durante las elecciones presidenciales podría entenderse en función de la frecuencia de la participación, por cuanto como se vio en el Gráfico 2, la mayoría de los encuestados indicó que había participado de 1 a 3 ocasiones.

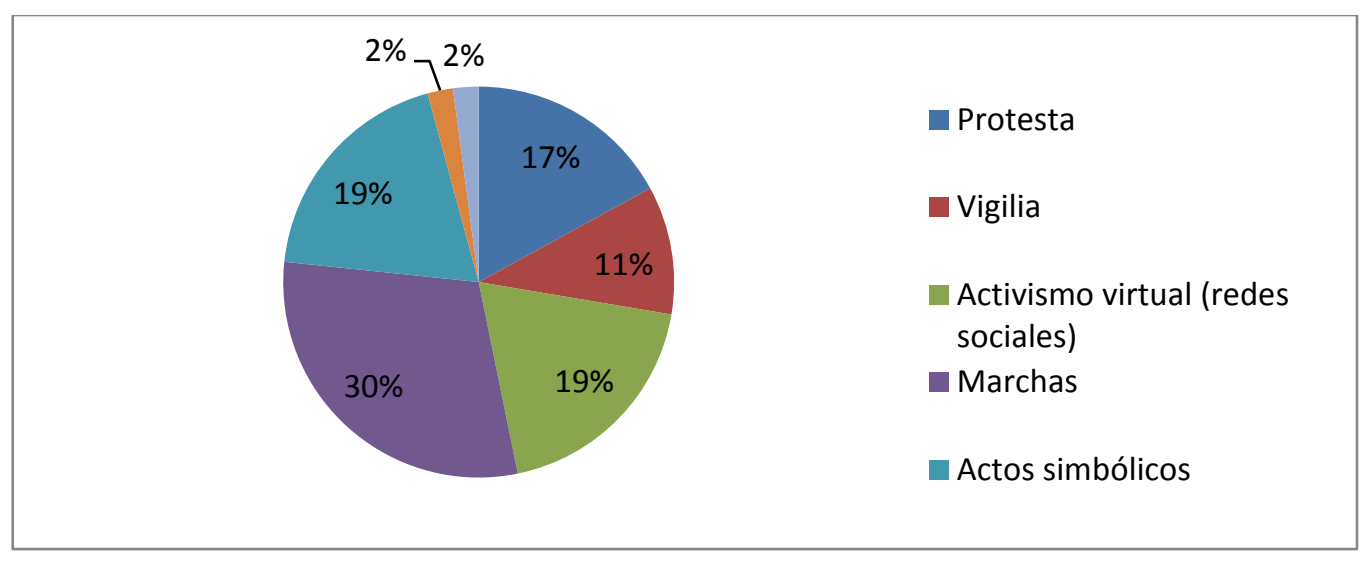

Gráfico 3: Mecanismos de participación

Fuente: Encuesta sobre no violencia a estudiantes universitarios Elaborado por: Paola Lozada

Finalmente, para indagar sobre el nivel de organización de la participación juvenil durante las elecciones presidenciales se realizaron dos preguntas, la primera sobre la existencia de grupos organizados y la segunda sobre la presencia de reglas o códigos que dirijan el actuar de los jóvenes. En cuanto al conocimiento de grupos organizados, el 53\% sabía de la existencia de grupos juveniles, no obstante, al analizar de qué grupos se trataban, se encontró que se referían tanto a los grupos juveniles promovidos por la Alianza Creo - Suma como a los grupos de Alianza País. Esta información permite reforzar la premisa de Sharp (2005) acerca de que la no violencia está identificada por lo que gente hace y no por lo que piensa, pues grupos de ambas tendencias políticas formaron parte de la campaña no violenta para transparentar los resultados.

A la pregunta sobre la presencia de reglas, códigos y similares que permitan dirigir la participación de los jóvenes, la mayoría de los encuestados reconoció su ausencia (73\%), mientras que aquellos que aseveraron contar con dichos elementos, se limitaron a señalar la exclusión de la violencia y no dejarse provocar.

En relación a estos resultados, aquellos jóvenes que sí registran una participación no violenta durante el proceso electoral, un tercio de los encuestados, la investigación explicaría que en virtud de la experiencia de otras luchas sociales en la que se ha visto inmiscuida la sociedad ecuatoriana y particularmente la quiteña durante el periodo presidencial de Rafael Correa, los jóvenes hayan optado por mecanismos conocidos como las marchas. Por otra parte, el 
incremento en el uso de las redes sociales entre la población de más de 12 años da cuenta de que uno de los mecanismos de participación preferidos por los jóvenes sea el activismo virtual.

Finalmente, el simbolismo como parte de la protesta social se privilegiaría debido a que facilita la identificación con un grupo específico, en este caso el no violento, provee de una forma de representación colectiva, y apoya la construcción de valores a nivel colectivo como la transparencia.

\section{Conclusión}

En función de los resultados de las encuestas la hipótesis se cumple parcialmente, pues si bien no se muestra una tendencia mayoritaria a la participación en la primera y segunda vuelta electoral, se encontró que el grupo de estudiantes encuestados no tiene una participación activa en las luchas sociales y conocen vagamente sobre la no violencia, variables que estarían estrechamente vinculadas, debido entre otras razones, a que los jóvenes carecen de la información y espacios propios de discusión tanto de los temas que aquejan a la sociedad, como de no violencia; en particular, sobre la organización, diversidad de mecanismos no violentos, códigos de conducta y la efectividad de su uso.

Adicionalmente, a pesar de la escasa participación juvenil durante el proceso electoral, los encuestados apoyan el hecho que los jóvenes se inmiscuyan en las luchas sociales, lo que mostraría la importancia y el interés que otorga dicho grupo poblacional a defender la justicia y practicar la democracia.

La campaña no violenta tuvo resultados significativos, pues si bien aún queda mucho trabajo con respecto a la educación en no violencia, permitió que los jóvenes reparen sobre la aplicabilidad de la no violencia en un contexto específico; adviertan sus alcances en la medida que el pueblo se una a la lucha; se interesen por temas que los afectarían directamente, al tratarse de la elección del presidente que regirá hasta el 2020 en Ecuador; y, contribuyan, junto con el resto de actores, a brindar una solución pragmática: el reconteo, como un primer paso en aras de conciliar las diferencias en un país dividido.

\section{Bibliografía}

AFP (23 de febrero de 2017). Lenín Moreno 39,36\% - Guillermo Lasso 28,09\%, con 99,92\% de votos escrutados por el CNE. El Comercio. Recuperado de: http://www.elcomercio.com/actualidad/resultados-elecciones-ecuador-guillermolassoleninmoreno.html.

AP (5 de junio de 2017). Ecuador: Anticipan que habrá una segunda vuelta electoral. La prensa. Recuperado de: http://www.prensa.com/mundo/Ecuador-anticipan-segunda-vueltaelectoral_0_4695280494.html

Apertura a los acuerdos de comercio (10 de enero de 2017). El Comercio. Recuperado de: http://www.elcomercio.com/actualidad/candidatos-apertura-acuerdoscomercialeselecciones.html

Aquí todas las revelaciones de Carlos Pareja Yannuzzelli con los episodios de CapayaLeaks (3 de febrero de 2017). El Universo. Recuperado de: 
http://www.eluniverso.com/noticias/2017/02/03/nota/6030187/aqui-toda-revelaciones-carlospareja-yannuzzelli-episodios

Basabe-Serrano S. (2014). Ecuador: Cada vez menos democracia, cada vez más autoritarismo... con elecciones. Revista de Ciencia Política. Volumen 34. 145-170.

Burrowes R. (1996). The Strategy of Nonviolent Defense: A Gandhian Approach. Albany, New York: State University of New York.

Cedatos (2017). Resultados de Exit poll de segunda vuelta para presidentes y vicepresidentes Elecciones 02 de abril de 2017. Recuperado de: https://www.cedatos.com.ec/detalles_noticia.php?Id=313

Cedatos (5 de abril de 2017). Cedatos anuncia a la opinión pública un nuevo atentado contra sus sistemas de información. Recuperado de: https://www.cedatos.com.ec/detalles_noticia.php?Id=326

CNE (10 de enero de 2017). La Escuela Politécnica Nacional se encargará de la parte técnica para conteo rápido de las Elecciones Generales 2017. Recuperado de: http://cne.gob.ec/es/institucion/sala-de-prensa/noticias/3850-la-escuela-politecnica-nacionalse-encargara-de-la-parte-tecnica-para-conteo-rapido-de-las-elecciones-generales-2017

CNE cerró centro de transmisión de resultados antes de medianoche (20 de febrero de 2017). El Universo. Recuperado de: http://www.eluniverso.com/noticias/2017/02/20/nota/6056701/cnecerro-centro-transmision-resultados-antes-medianoche

Conaie (2017). La Conaie frente a los acontecimientos electorales. Recuperado de: https://conaie.org/2017/02/21/la-conaie-frente-los-acontecimientos-electorales/

Consejera del CNE anunció apoyo a un recuento de votos "bien fundamentado" (5 de abril de 2017). El Universo. Recuperado de: http://www.eluniverso.com/noticias/2017/04/05/nota/6125222/consejera-cne-anuncio-apoyoreconteo-bien-fundamentado

Consejo de Generales del Ejército (2017). El Ejército Ecuatoriano al país. Recuperado de: http://www.elcomercio.com/actualidad/consejo-generales-ejercito-respeto-elecciones.html

Creo denuncia irregularidades en1.795 actas que significan casi 600 mil votos (5 de abril de 2017). El Universo. Recuperado de: http://www.eluniverso.com/noticias/2017/04/05/nota/6124706/guillermo-lasso-anunciaimpugnacion-datos-anunciados-cne

El jefe saliente del Ejército llama a custodiar comicios (7 de marzo de 2017). El Universo. Recuperado de: http://www.eluniverso.com/noticias/2017/03/07/nota/6077269/jefe-saliente-ejercitollama-custodiar-comicios

Entrega de resultados del conteo rápido. Participación Ciudadana. Recuperado de: https://www.participacionciudadana.org/pc-entrega-resultados-del-conteo-rapido-candidatospresidenciales/ 
Exit poll da como vencedor de balotaje en Ecuador a Lenín Moreno ( 2 de abril de 2017). El Telégrafo. Recuperado de: http://www.eltelegrafo.com.ec/noticias/politiko-2017/49/exit-poll-da-comovencedor-de-balotaje-en-ecuador-a-lenin-moreno

Expectativa y desgaste en las protestas frente al CNE en Quito (6 de abril de 2017). El Universo. Recuperado de: http://www.eluniverso.com/noticias/2017/04/06/nota/6126515/expectativadesgaste-protestas-frente-cne-quito

Fuerzas Políticas resolverán apoyo para la segunda, tras resultados (21 de febrero de 2017). El Universo. Recuperado de: http://www.eluniverso.com/noticias/2017/02/21/nota/6057961/fuerzas-politicas-resolveranapoyo-segunda-tras-resultados

Galo Chiriboga y presidente tienen familiares en común (3 de mayo de 2011). El Universo. Recuperado de: http://www.eluniverso.com/2011/05/03/1/1355/galo-chiriboga-presidente-tienenfamiliares-comun.html

García, J. (2015). E-commerce Day. Instituto Nacional de Estadísticas y Censos. Recuperado de: http://www.ecuadorencifras.gob.ec/documentos/web-inec/boletin/E-commerce.pdf

Helvey, R. (2004). On Strategic Nonviolent Conflict: Thinking about the Fundamentals. Boston: Albert Einstein Institution.

Increase of the criminalization of social protest in the context of extraction plans (10 de diciembre de 2015). FIDH. Recuperado de: https://www.fidh.org/en/region/americas/ecuador/ecuadorincrease-of-the-criminalisation-of-social-protest-in-the

INEC (2010). Censo de Población y Vivienda. Recuperado de: http://www.ecuadorencifras.gob.ec/base-de-datos-censo-de-poblacion-y-vivienda/

Informe Anual 2015 de la Relatoría Especial para la libertad de expresión, Recuperado de: http://www.oas.org/es/cidh/expresion/docs/informes/anuales/InformeAnual2015RELE.pdf

Lasso presentó red de control electoral para cuidar voto ciudadano (14 de febrero de 2017). Movimiento Creo. Recuperado de: http://creo.com.ec/noticias/lasso-presento-red-de-controlelectoral-para-cuidar-voto-ciudadano/

Lasso y su familia fueron agredidos a la salida del estadio Atahualpa; en redes se rechazó la violencia (28 de marzo de 2017). El Comercio. Recuperado de: http://www.elcomercio.com/actualidad/guillermolasso-familia-ataque-exterioresolimpicoatahualpa.html

Ley Orgánica Electoral y de Organizaciones Políticas de la República del Ecuador Código de la Democracia. Registro Oficial Suplemento 578, Ecuador, 27 de abril de 2009.

Megale, B., Mattoso, C., Wiziack, J., \& Valente, R. (21 de diciembre de 2016). Grupo Odebrecht pagou US\$ 1 bi em propinas em 12 países, dizem EUA. Folha de S. Paulo. Recuperado de: http://www1.folha.uol.com.br/poder/2016/12/1843417-odebrecht-pagou-us-788-milhoes-empropinas-em-12-paises-dizem-eua.shtml 
Mena, P. (14 de abril de 2014). "Yasunidos", los jóvenes que desafían a Correa en la polémica por Yasuní. BBC. Recuperado de: http://www.bbc.com/mundo/noticias/2014/04/140414_ecuador_petroleo_parque_yasuni_mxa

Miller, C. $\left(2006^{a}\right)$. Strategic Nonviolent Struggle: A Training Manual. Nonviolent Transformation of Conflict-Africa Series. Addis Ababa and Geneva: University for Peace.

Miller, C. (2006b). Only Young Once: An Introduction to Nonviolent Struggle for Youths. Nonviolent Transformation of Conflict--Africa Series. Addis Ababa and Geneva: University for Peace.

Movimiento País (2014). Nuestras 10 Revoluciones. Recuperado de: http://www.alianzapais.com.ec/2016/07/08/nuestras-10-revoluciones/

Movimiento País (2016). Programa de Gobierno. Recuperado de: http://www.alianzapais.com.ec/wpcontent/uploads/2016/11/prog_gob_revista.pdf

Noboa A. (3 de abril de 2017). CNE sobre problemas técnicos "Sistema nunca se cayó ni dejó de funcionar". El Comercio. Recuperado de: http://www.elcomercio.com/actualidad/cneproblemas-sistema-caida-resultados.html

Ochoa, C. (5 de abril de 2017). Superintendencia de la Información y la Comunicación. Recuperado de: http://www.supercom.gob.ec/es/sala-de-prensa/noticias/1398-supercom-leycomunicacion-radio-vision-llamado-atencion-carlosochoa

Oficinas de Cedatos y una agencia, allanadas por supuesta alteración (8 de abril 2017). El Comercio. Recuperado de: http://www.elcomercio.com/actualidad/oficinas-cedatos-allanamientofiscalia-policia.html

Pablo Ospina, “¿Por qué protestan en Ecuador? Rafael Correa y el fracasado aumento del impuesto a las herencias". Nueva Sociedad N²58, (julio- agosto 2015).

Pallares, M. (20 de marzo de 2017). La Mofle gritó ¡Fraude! Y la censuraron en Gama TV. Cuatro Pelagatos. Recuperado de: http://4pelagatos.com/2017/03/20/la-mofle-censurada-porque-suschistes-no-hacen-reir-al-gobierno/

Palomeque dice que la vetaron por decir "fraude" en Gama TV (21 de marzo de 2017). La Hora. Recuperado de: http://lahora.com.ec/index.php/noticias/show/1102041065/1/Palomeque_dice_que_la_\%E2\%80\%98vetaron\%E2\%80\%99_por_decir_\%E2\%80\%98frau de\%E2\%80\%99_en_Gama_TV.html\#.WTmGaes1_IV

Participación Ciudadana (2 de abril de 2017). Participación Ciudadana entre de resultados del conteo rápido candidatos presidenciales segunda vuelta. Recuperado de: https://www.participacionciudadana.org/participaci\%C3\%B3n-ciudadana-entrega-resultadosdel-conteo-r\%C3\%A1 pido-candidatos-presidenciales-segunda-vuelta/

Participación Ciudadana (2017). Elección de Presidente y Vicepresidente - 1era vuelta electoral. Recuperado de: https://www.participacionciudadana.org/elecciones-2017/ 
Pativideo sembró dudas sobre una manipulación (30 de abril de 2011). El Comercio. Recuperado de: http://www.elcomercio.com/actualidad/mundo/pativideo-sembro-dudas-manipulacion.html

Presidencia de la República del Ecuador (8 de abril de 2017. Enlace Ciudadana N ${ }^{\circ} 518$ desde Palenque - Los Ríos. Recuperado de: http://www.enlaceciudadano.gob.ec/enlace-ciudadano-no-518desde-palenque-los-rios/

Recuento de votos ratifica la victoria de Lenín Moreno en balotaje en Ecuador (18 de abril de 2017). El Telégrafo. Recuperado de: http://www.eltelegrafo.com.ec/noticias/politiko-2017/49/fuerteresguardo-militar-y-policial-para-el-recuento-de-votos

Romero, D. (19 de febrero de 2017). La policía aclaró que hubo cinco papeletas marcadas en cinco cantones. El Comercio. Recuperado de: http://www.elcomercio.com/actualidad/policiapapeletasmarcadas-denuncias-elecciones.html

Schock, K. (2005). In Unarmed Insurrections: People Power Movements in Nondemocracies. Minneapolis and London: University of Minnesota Press.

Sharp,

G. (2005). http://www.elearning.upeace.org/classroom/pluginfile.php/8628/course/section/1282/Sharp_ Gene_25-38_2.pdfWaging Nonviolent Struggle: 20th Century Practice and 21st Century Potential. Boston: Porter Sargent.

Sistema Integrado de Conocimiento y Estadística Social del Ecuador - SICES (2016). Tasa bruta de matrícula en educación superior. Recuperado de: http://www.conocimientosocial.gob.ec/pages/EstadisticaSocial/herramientas.jsf 